ACTA UIVERSITATIS CIBINIENSIS - TECHNICAL SERIES

Vol. LXVIII 2016

\title{
Dual solution of Casson fluid over a porous medium: Exact solutions with extra boundary condition
}

\author{
Najeeb Alam Khan ${ }^{*}$, Sidra Khan \\ Department of Mathematics, University of Karachi, Karachi 75270, Pakistan
}

\begin{abstract}
In this article we calculate the exact solution of the steady flow of non-Newtonian Casson fluid, over a stretching/shrinking sheet. The governing partial differential equations (PDEs) are transformed into ordinary differential equation (ODE) by using similarity transformation and then solved analytically by utilizing the exact solution. The closed form unique solution is obtained in the case of stretching sheet whereas for shrinking sheet unique and dual solutions are obtained. Influences of Casson fluid and suction/injection parameter on dimensionless velocity function are discussed and plotted graphically; also the effects of skin friction coefficient are presented in graphical form. Comparisons of current solutions with previous study are also made for the verification of the present study.
\end{abstract}

Key words: Casson fluid; stretching/shrinking; exact solution

\section{Introduction:}

The study of non Newtonian fluid captivated the interest of new researchers due to their increasing practical relevance in different fields of engineering and industry. Several researchers have worked on different Newtonian and non-Newtonian fluid models, among which the nonNewtonian Casson fluid has excited more concentration of researchers due to its applications in different industrial fields like, drilling operations, food processing, metallurgy, and bioengineering operations. This Casson fluid model is also preferred for the examination of human blood due to its constituents like red cells, plasma, protein, etc. Some daily life fluids like jelly, soup, tomato paste and honey also relate the rheological properties of Casson fluid [1]. Recently many new researchers have considered the flow of Casson fluid in different geometries like, Ramesh and Devakar [2] examined the three models (Couette, Poiseuille, and generalized Couette flows) with slip conditions, Mythili and Sivaraj [3] studied Casson flow over a flat plate and vertical cone with the effects of temperature, chemical reaction and thermal radiation. Hakeem et al. [4] analyzed the inclined MHD flow of Casson fluid over an impermeable stretching sheet in the presence of heat transfer. Abbas et al. [5] calculated the numerical solution of unsteady Casson boundary layer flow close to stagnation point over stretching/shrinking sheet with heat and mass transfer effect. Kataria and Patel [6] investigated the unsteady free convective hydromagnetic boundary layer Casson fluid flow past an oscillating vertical plate implanted through porous medium with chemical, thermal and uniform transverse magnetic field effects. Raju et al. [7] calculated the MHD flow of non-Newtonian Casson model over an exponentially permeable stretching surface with thermal radiations and chemical reaction. 
The boundary layer flow of Newtonian and non-Newtonian fluids over stretching/shrinking sheet have attained the attention of researchers because of their industrial applications such as metal spinning, paper production, wire drawing, hot rolling, glass-fiber production, polymer processing, etc. Most of the works have been done with the non-Newtonian fluid models like; Bhattacharyya et al. [8] investigated the exact solution of boundary layer flow of two dimensional Casson fluid over a stretching/shrinking sheet in the presence of porous mass transfer effect. Pramanik [9] studied the effect of Casson fluid flow towards an exponentially porous stretching surface with thermal radiation, Mythili and Sivaraj [10] calculated the influence of Casson fluid over a flat plate and vertical cone with non uniform heat source/sink and chemical reaction. Mansur and Ishak [11] examined the impact of boundary layer flow of nanofluids over a stretching/shrinking sheet through a convective surface boundary condition. Mohanty et al. [12] presented the effect of an unsteady, incompressible micropolar fluid over a stretching sheet through porous medium with heat and mass transfer characteristics.

The present paper is based on the investigation of steady boundary layer flow of nonNewtonian Casson fluid over a permeable stretching/shrinking sheet with and without mass transfer effect. The governing equations are transformed using similarity transformation and solved analytically. From all cases we obtained the closed form exact solution except shrinking sheet case considered without suction/injection. The effect of Casson fluid parameter and suction/injection parameter are discussed and presented in graphical form, also the comparison has been made with the previous study [8] for the verification of the current solutions.

\section{Problem Formulation}

Consider the flow of steady, incompressible two dimensional Casson fluid over a stretching/shrinking sheet. The sheet is stretched along $x$-axis at $y=0$ and fluid is placed along the $y$-axis $y>0$. Fig. 1 represents the geometrical configuration of the physical model. Here we consider that stretching sheet has a linear velocity $U=b x$, along $x$-axis respectively with constant $b$. The rheological equation of Casson fluid is written as Eq. (1) where $\tau$ is extra stress tensor for an isotropic flow shown as [8]

$$
\tau_{i j}=\left\{\begin{array}{l}
\left(\mu_{B}+\frac{p_{y}}{\sqrt{2 \pi}}\right) 2 e_{i j}, \quad \pi>\pi_{c} \\
\left(\mu_{B}+\frac{p_{y}}{\sqrt{2 \pi_{c}}}\right) 2 e_{i j}, \quad \pi<\pi_{c}
\end{array}\right.
$$

Where $\mu$ is the dynamic viscosity, $\mu_{B}$ is a plastic dynamic viscosity of non-Newtonian fluid, $p_{y}$ is the yield stress of fluid, $\pi$ is the product of the component of deformation rate with itself, namely, $\pi=e_{i j} e_{i j}, e_{i j}$ is the $(i, j)$-th component of the deformation rate and $\pi_{c}$ is critical value of this product based on non-Newtonian model 
The boundary layer approximation of the problem is determined by the following equations:

$\frac{\partial u}{\partial x}+\frac{\partial v}{\partial y}=0$

$u \frac{\partial u}{\partial x}+v \frac{\partial u}{\partial y}=-\frac{p_{x}}{\rho}+v\left(1+\frac{1}{\beta}\right) \frac{\partial^{2} u}{\partial y^{2}}$

where the velocity components $(u, v)$ are in the $(x, y)$ directions, respectively. The Casson fluid parameter is $\beta=\mu_{B} \sqrt{2 \pi_{c}} / p_{y}$. Here $p_{x}=0$, due to the motion of fluid, which is caused by dragging the fluid along with the moving boundary.

$u(x, 0)-U=v\left(1+\frac{1}{\beta}\right) \frac{\partial u}{\partial y}(x, 0)$

where $v$ is the kinematic viscosity of the fluid.

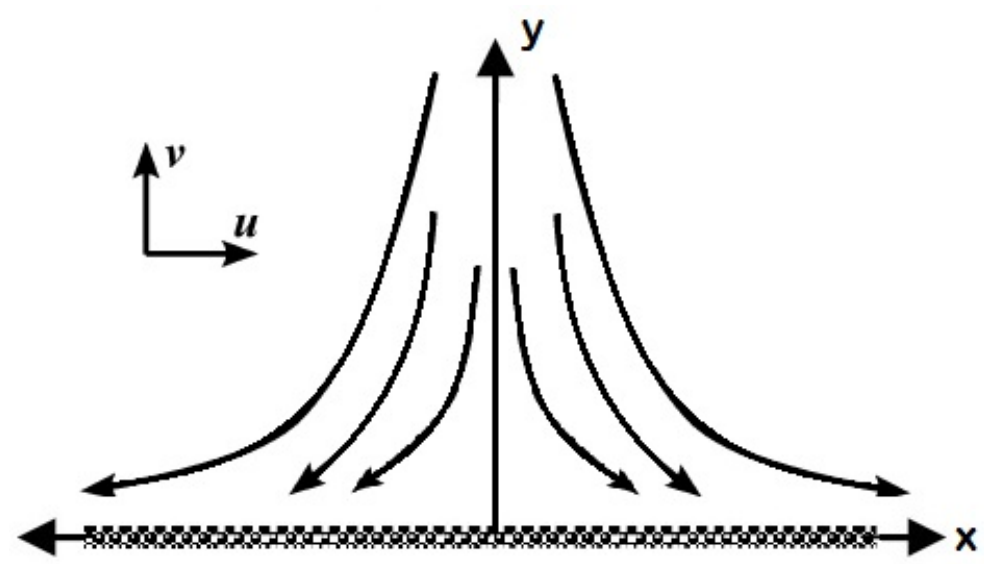

Fig 1: Physical model and coordinate system.

From [13] it is shown that the values of $v$ component at $a \rightarrow \infty$ is,

$v(y)=\frac{-y Q}{2 \pi a^{2}}$

where we have considered a case of source of intensity $Q$ in front of a wall in $x$ direction. Note that $v$ is a function of $y$ and $v<0[14]$. 
Using the above argument as a guide, we define $f(\eta)$ as,

$v=-f(\eta)$

where $f(\eta) \geq 0,0 \leq \eta<\infty$ and

$\eta=y \sqrt{\frac{b}{v}}$

From Eq. (2) and Eq. (6) we figure out that

$u_{x}=-v_{y} \Rightarrow f_{y}(\eta)=\sqrt{\frac{b}{v}} f^{\prime}(\eta)$

that is

$u_{x}=\sqrt{\frac{b}{v}} f^{\prime}(\eta)$

where prime denotes differentiation with respect to $\eta$.

Since $f=f(\eta)$, after integrating Eq. (9), we get

$u=\sqrt{\frac{b}{v}} f^{\prime}(\eta) x$

(by choosing an arbitrary function of $\eta$,zero).

The equation of motion is simplified by using the following constant of proportionality into Eq. (6)

$v=-\sqrt{b v} f(\eta)$

hence, Eq. (10) is revise as

$u=b f^{\prime}(\eta) x$

Thus, by utilizing the equations (11), (12), and (7), equation of continuity satisfied. By means of Eqs. (7), (11) and (12), Eq. (3) can be rewritten as

$u b f^{\prime}(\eta)+\sqrt{\frac{b}{v}} b v x f^{\prime \prime}(\eta)-\left(1+\frac{1}{\beta}\right) b^{2} x f^{\prime \prime \prime}(\eta)=0$ 
by utilizing the Eq. (11) and Eq. (12) into Eq. (13), we attain

$$
\left(1+\frac{1}{\beta}\right) f^{\prime \prime \prime}-f^{\prime 2}+f f^{\prime \prime}=0,
$$

To conclude the boundary conditions of Eq. (14), we see that $v(y=0)=0$; from Eq. (7) and Eq. (11) we obtain

$$
f(0)=0
$$

in the similar manner, from the condition $\lim _{y \rightarrow \infty} u(x, y)=0$, and (7), we attain the following boundary condition

$f^{\prime}(\infty)=0$

For condition $f^{\prime}(\infty) \rightarrow 0$; we assume an extra boundary condition

$$
f(\infty) \rightarrow \xi
$$

where $\xi$ is any constant.

To conclude, by substituting linear velocity of stretching sheet and Eq. (12) into Eq. (2), we find

$$
b x f^{\prime}(0)-b x=0
$$

the above equation reduces as

$$
f^{\prime}(0)=1
$$

\section{Exact solution}

In this section 3, the handy exact solutions of two different cases are calculated. In first case, we have considered the case without mass transfer parameter; and in second case, we have considered the case with mass transfer parameter.

\subsection{Case 1}

Consider the equation (14) solving with the boundary conditions Eq. (15)-(17) without mass transfer parameter $S=0$.

Rewriting (14) in the form

$$
f=\frac{f^{\prime 2}-\alpha f^{\prime \prime \prime}}{f^{\prime \prime}},
$$


Where $\alpha=1+\frac{1}{\beta}$ is a fluid parameter and by using boundary condition (17), Eq. (20) lead as

$$
\xi f^{\prime \prime}=-\alpha f^{\prime \prime \prime}
$$

Squaring both the sides of Eq. (21), and rewrite as

$$
\xi^{2} f^{\prime \prime 2}=\alpha^{2} f^{\prime \prime \prime 2}
$$

Defining new variable

$$
F=f^{\prime}
$$

Eq. (23) will become

$$
\xi^{2} F^{2}=\alpha^{2} F^{\prime \prime 2}
$$

Similarly, by using $F=f^{\prime}$ it is possible to express Eq. (20) as

$$
f=\frac{F^{2}-\alpha F^{\prime \prime}}{F^{\prime}},
$$

Differentiating Eq. (25) as follows

$$
F=\frac{F^{\prime}\left(2 F F^{\prime}-\alpha F^{\prime \prime \prime}\right)-F^{\prime \prime}\left(F^{2}-F^{\prime \prime}\right)}{F^{\prime 2}}
$$

Equation (25) and (24) take the following limit forms when $F(\infty) \rightarrow 0$ (boundary condition Eq. (16)) and boundary condition (17).

$$
\begin{aligned}
& \alpha F^{\prime \prime}+\xi F^{\prime}=0 \\
& F^{\prime \prime 2}-F^{\prime} F^{\prime \prime \prime}=0
\end{aligned}
$$

Also from Fig. 1 and Eq. (11) it follows that $v<0$ and $\xi>0$.

$$
F^{\prime \prime \prime}-\frac{\xi^{2}}{\alpha^{2}} F^{\prime}=0
$$

In order to solve Eq. (29) we have suppose the new variable

$$
z=F^{\prime}
$$

which reduced the order of equation as 
$z^{\prime \prime}-\frac{\xi^{2}}{\alpha^{2}} z=0$

The above Eq. (31) is second order homogenous equation, which has the solution

$z=C_{1} e^{\frac{\xi}{\alpha} \eta}+C_{2} e^{-\frac{\xi}{\alpha} \eta}$

Where $C_{1}$ and $C_{2}$ are constants. To avoid that $z \rightarrow \infty$, when $x \rightarrow \infty$ (from Eq. (16), (23), and (30) is clear that for that limit), we choose $C_{1}=0$ so that Eq. (32) adopts the simpler form

$$
z=C_{2} e^{-\frac{\xi}{\alpha} \eta}
$$

Also from Eq. (30) and Eq. (33) we obtain

$$
F^{\prime}=C_{2} e^{-\frac{\xi}{\alpha} \eta}
$$

After integrating the above Eq. (34) lead as

$$
F=-\frac{\alpha C_{2}}{\xi} e^{-\frac{\xi}{\alpha} \eta}+c_{1}
$$

By solving Eq. (35) using boundary condition $F(\infty) \rightarrow 0$, integration constant $c_{1}=0$.

$$
F=-\frac{\alpha C_{2}}{\xi} e^{-\frac{\xi}{\alpha} \eta}
$$

Integrating again, we get

$$
f=\frac{\alpha^{2} C_{2}}{\xi^{2}} e^{-\frac{\xi}{\alpha} \eta}+c_{2}
$$

Solving Eq. (37) by using boundary condition Eq. (15); $c_{2}=-\frac{\alpha^{2} C_{2}}{\xi^{2}}$

$$
f=\frac{\alpha^{2} C_{2}}{\xi^{2}}\left(e^{-\frac{\xi}{\alpha} \eta}-1\right)
$$

Using second boundary condition Eq. (17); $C_{2}=\frac{-\xi \xi^{2}}{\alpha^{2}}$ which lead Eq. (38) as 
$f=\xi\left(1-e^{-\frac{\xi}{\alpha} \eta}\right)$

\subsubsection{Stretching Sheet}

To find out the value of $\xi$, we used the stretching sheet conditions

$f^{\prime}(0)=1$

Using Eq. (40) to solve Eq. (39),

$f^{\prime}=\frac{\xi^{2}}{\alpha} e^{-\frac{\xi}{\alpha} \eta} \quad$ at $f^{\prime}(0)=1$

By solving Eq. (41), we obtain the value of $\xi= \pm \sqrt{\alpha}$. As $e^{-\frac{\xi}{\alpha} \eta}$; to converge, we need positive value $\xi=+\sqrt{\alpha}$ as solution

$f=\sqrt{\alpha}\left[1-e^{-\frac{\sqrt{\alpha}}{\alpha} \eta}\right]$

Substituting the value of $\alpha$ in Eq. (42), we obtain

hence $f=\sqrt{\left(1+\frac{1}{\beta}\right)}\left[1-e^{\left.-\frac{\eta}{\sqrt{\left(1+\frac{1}{\beta}\right)}}\right]}\right.$.

\subsubsection{Shrinking Sheet}

No solution exists for shrinking sheet, as the condition is

$f^{\prime}(0)=-1$

So that by using the boundary condition Eq. (43) in Eq. (40) we obtain the value of $\xi$ as

$$
\xi= \pm \sqrt{-\alpha}
$$

Because of the negative sign inside radical, the condition is not a real one. Thus, the solution does not exist on real axis.

\subsection{Case 2}


In this case two, we are using the same problem with mass transfer parameter $S \neq 0$ for solving the differential equation (31) which gives us a solution Eq. (37).

\subsubsection{Stretching Sheet}

Considering the boundary conditions of stretching sheet are,

$f(\eta)=S, f^{\prime}(\eta)=1$ at $\eta=0$

$f(\eta)=\xi, f^{\prime}(\eta)=0 \quad$ at $\eta=\infty$

Using the same procedure as above, considering the boundary conditions Eq. (45) and Eq. (46), we get the solution of ordinary differential equation (31) as

$$
\begin{aligned}
f(\eta)= & S \exp \left[-\frac{\left(S+\sqrt{S^{2}-4 \alpha}\right)}{2 \alpha} \eta\right]-\frac{\left(S+\sqrt{S^{2}-4 \alpha}\right)}{2} \exp \left[-\frac{\left(S+\sqrt{S^{2}-4 \alpha}\right)}{2 \alpha} \eta\right] \\
& +\frac{\left(S+\sqrt{S^{2}-4 \alpha}\right)}{2}
\end{aligned}
$$

where $\xi=\frac{\left(S+\sqrt{S^{2}-4 \alpha}\right)}{2}$

\subsubsection{Shrinking Sheet}

Considering the boundary conditions of shrinking sheet are,

$f(\eta)=S, f^{\prime}(\eta)=-1 \quad$ at $\eta=0$

$f(\eta)=\xi, f^{\prime}(\eta)=0 \quad$ at $\eta=\infty$

Using the same procedure as above, considering the boundary conditions Eq. (48) and Eq. (49), we get the solution of ordinary differential equation (31) as

$$
\begin{aligned}
\xi=\frac{S \pm \sqrt{S^{2}+4 \alpha}}{2} \\
f(\eta)=S \exp \left[-\frac{S \pm \sqrt{S^{2}+4 \alpha}}{2 \alpha} \eta\right]-\frac{S \pm \sqrt{S^{2}+4 \alpha}}{2} \exp \left[-\frac{S \pm \sqrt{S^{2}+4 \alpha}}{2 \alpha} \eta\right] \\
+\frac{S \pm \sqrt{S^{2}+4 \alpha}}{2 \alpha}
\end{aligned}
$$

\section{Results and Discussion}


The behavior of physical parameter for various values on case 1 and case 2 are shown graphically. Fig 2 shows the influence of skin friction coefficient $f^{\prime \prime}(0)$ when the fluid parameter $\beta$ varies against the mass transfer parameter $S$. It shows that magnitude of skin friction is directly proportional to fluid parameter, when $\beta$ decrease it also reduce the magnitude of skin friction coefficient. The Casson parameter is inversely proportional to the yield stress, so when $\beta$ start decreasing it increases the yield stress and consequently decreased the skin friction coefficient.

Fig. 3 and Fig. 4 demonstrate the effect of $\beta$ and $S$ on dimensionless velocity function $f^{\prime}(\eta)$ for stretching case. It is evident from figure 3 that increase in fluid parameter $\beta$ decreases the boundary layer thickness of velocity function $f^{\prime}(\eta)$ for suction $S=2$. The impact of suction and injection for stretching case with constant value $\beta=1$ shows that increasing values of suction $S=1,2$ shows thinner effect whereas different values of injection $S=-1,-2$ shows thicker effect on velocity function $f^{\prime}(\eta)$ (see figure 4).

The dual solutions of shrinking sheet flow of Casson fluid are shown in Fig. 5. The dual nature of similarity solution is presented in Fig. 5 for skin friction coefficient $f^{\prime \prime}(0)$ against $S$ for various values of fluid parameter $\beta$. For boundary layer flow of Casson fluid, strong wall mass suction is needed because decrease in Casson parameter enhance the stress and strengthen the vorticity formed by the shrinking sheet. For the maintenance of the boundary layer we control the vorticity present inside boundary layer by strong wall mass suction. At decreasing values of $\beta$, the fist solution of skin coefficient reduces and enhances in second solution.

The dual velocity profile $f^{\prime}(\eta)$ for various values of Casson fluid parameter $\beta$ is presented in Fig. 6 (when $S>0$ ). It is observed that the boundary layer thickness of velocity function decrease rapidly with increasing values of Casson parameter $\beta$ for the first solution whereas it shows opposite slight increasing behavior for the second solution. Fig. 7 demonstrates the effect of stream function $f(\eta)$ for fluid parameter $\beta$, which shows the same decreasing and increasing behavior for the first and second solution. 
The influence of suction parameter $S$ on dimensionless dual velocity profile $f^{\prime}(\eta)$ is manifest in Fig. 8, which expresses the similar pattern as we seen in Fig. 6 for fluid parameter $\beta$. Increasing values of suction parameter shows slight deceleration for the first solution whereas solution two shows a rapid acceleration in the boundary layer thickness of velocity function $f^{\prime}(\eta)$. Fig.9 and Fig. 10 shows the comparison of present study with the previous study [8], which shows a promising agreement.

\section{Conclusions}

A steady, incompressible flow of non-Newtonian Casson fluid over a porous medium (stretching/shrinking sheet) is studied. Analytical closed formed unique solutions is attained for stretching sheet cases 1, whereas shrinking sheet doesn't give us a real solution because of negative sign inside radical. The closed form unique solution is obtained in Case 2 (stretching sheet with mass transfer effect) whereas unique as well as dual nature solutions are obtained from shrinking sheet for various values of fluid parameter and suction parameter but no similarity solution exist for injection values.

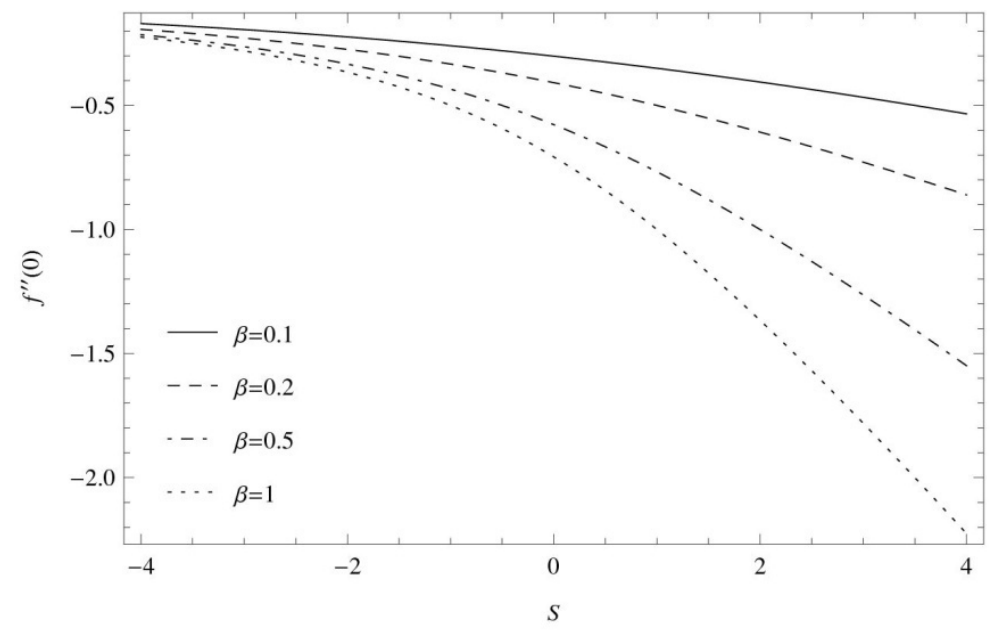

Fig. 2: Skin friction coefficient $f^{\prime \prime}(0)$ for several values of $\beta$ (stretching case) 


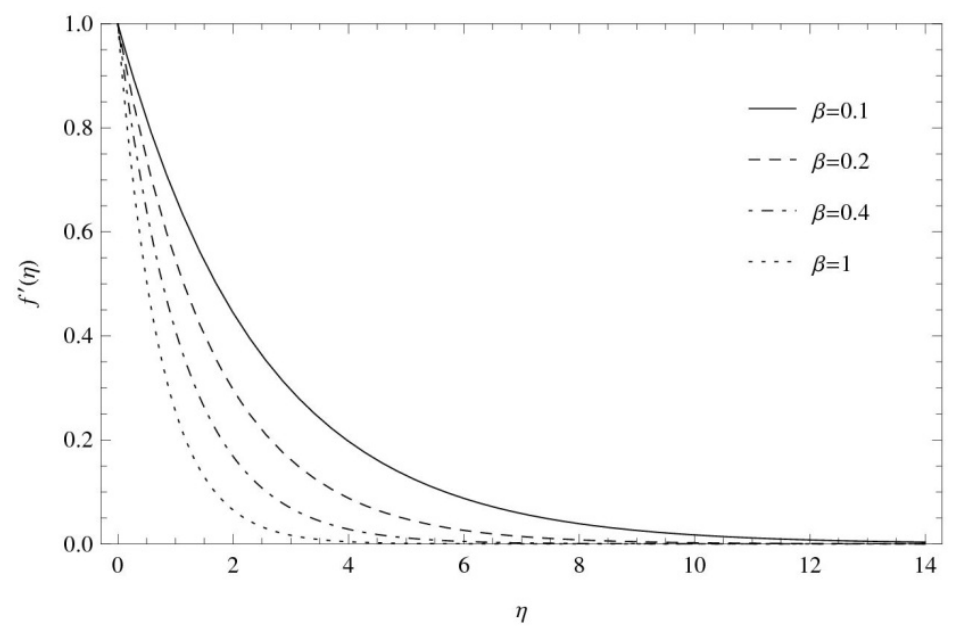

Fig. 3: Velocity profiles $f^{\prime}(\eta)$ for various values of $\beta$ (stretching case)

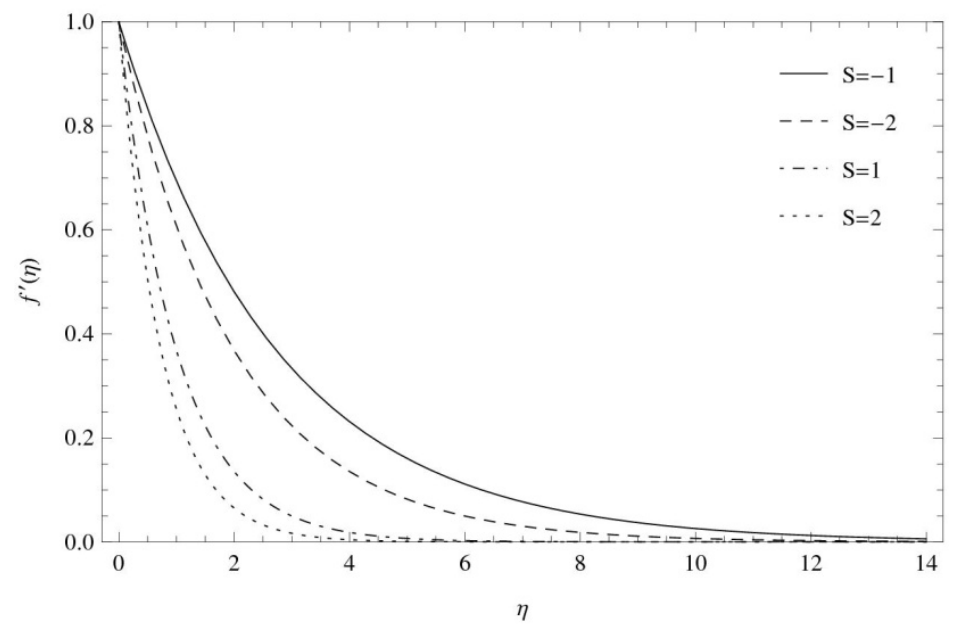

Fig. 4: Velocity profiles $f^{\prime}(\eta)$ for various values of $S$ (stretching case)

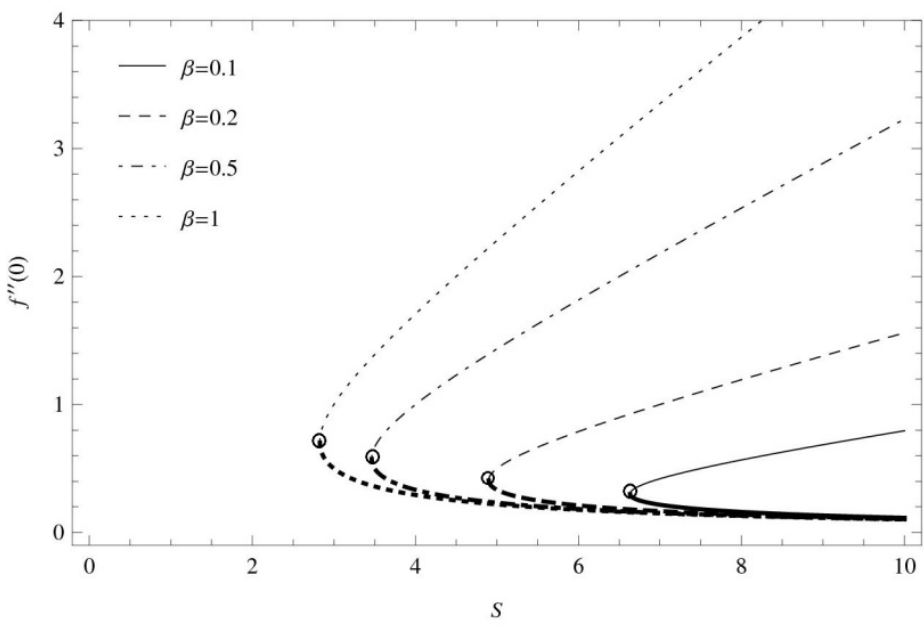


Fig. 5: Skin friction coefficient for several values of $\beta$ (shrinking case)

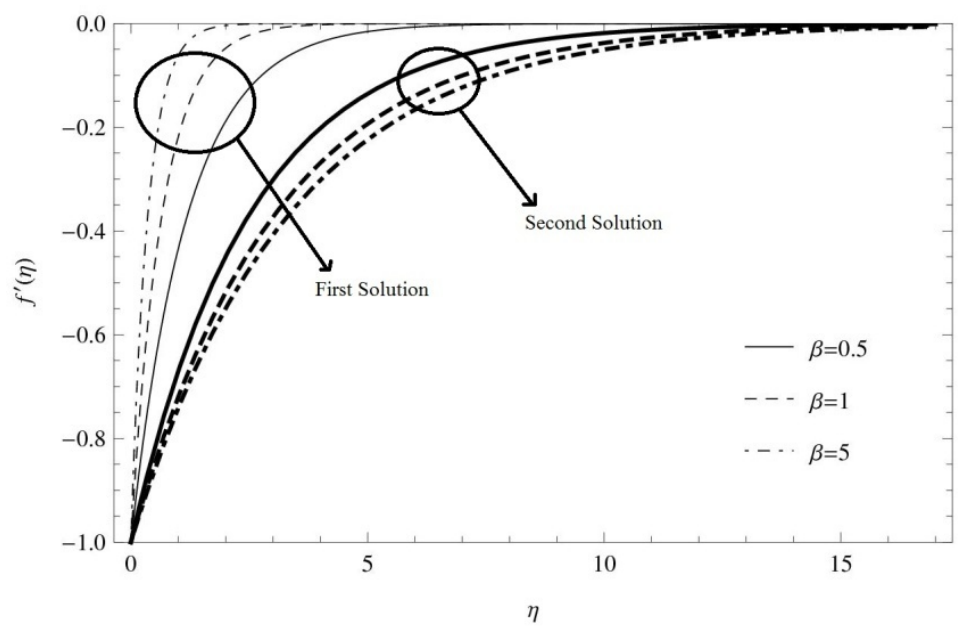

Fig. 6: Dual Velocity profiles $f^{\prime}(\eta)$ for various values of $\beta$ (shrinking case)

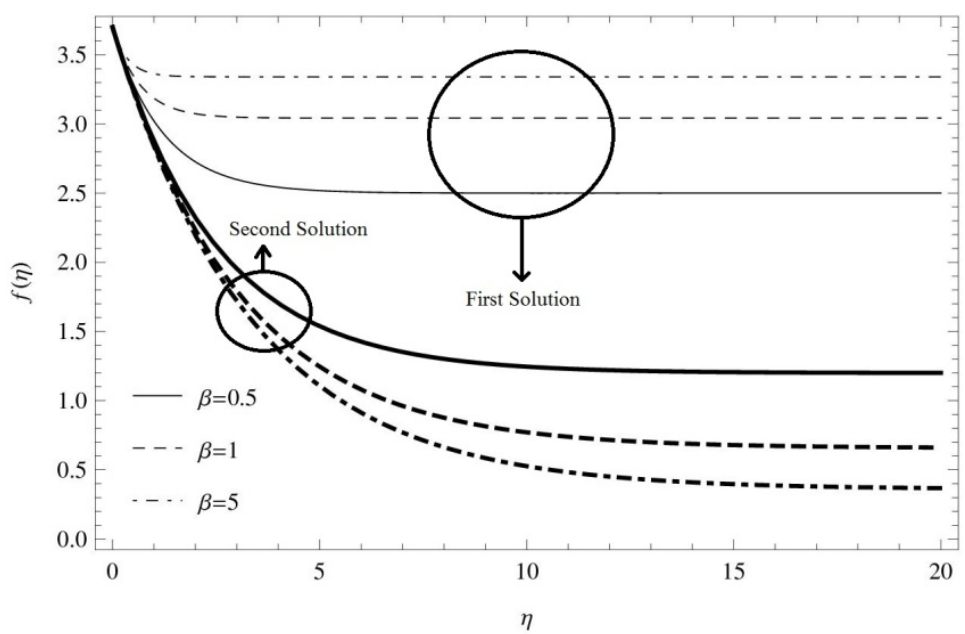

Fig. 7: Dimensionless stream function $f(\eta)$ for various values of $\beta$ (shrinking case) 


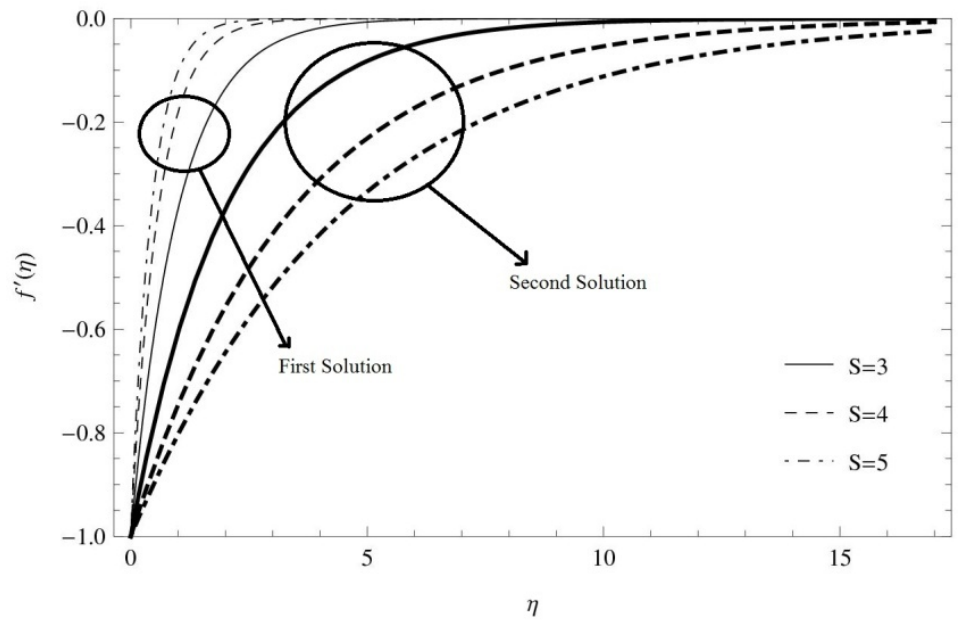

Fig. 8: Dual Velocity profiles $f^{\prime}(\eta)$ for various values of $S$ (shrinking case)

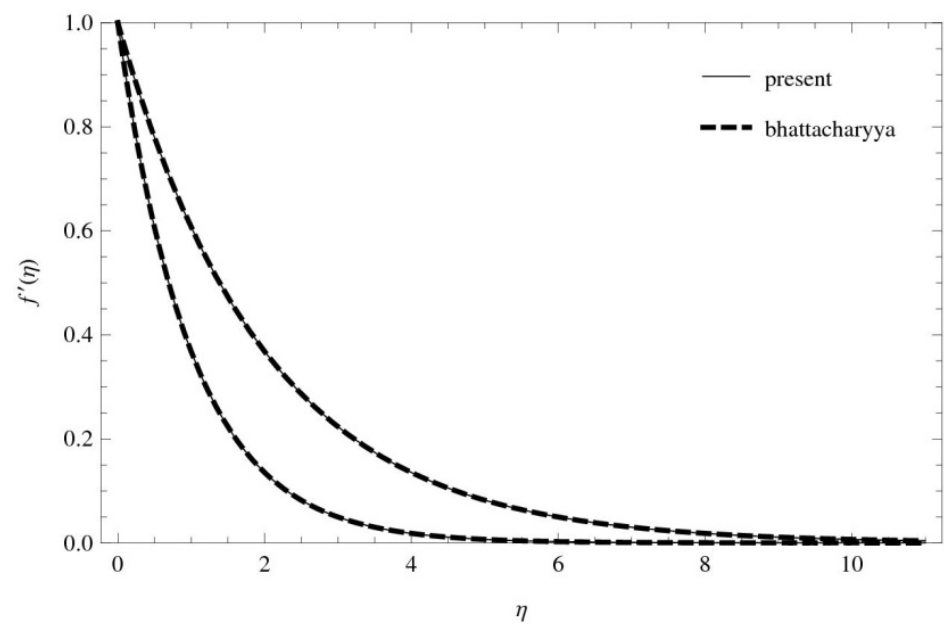

Fig. 9: Comparison of Velocity profiles $f^{\prime}(\eta)$ for stretching case

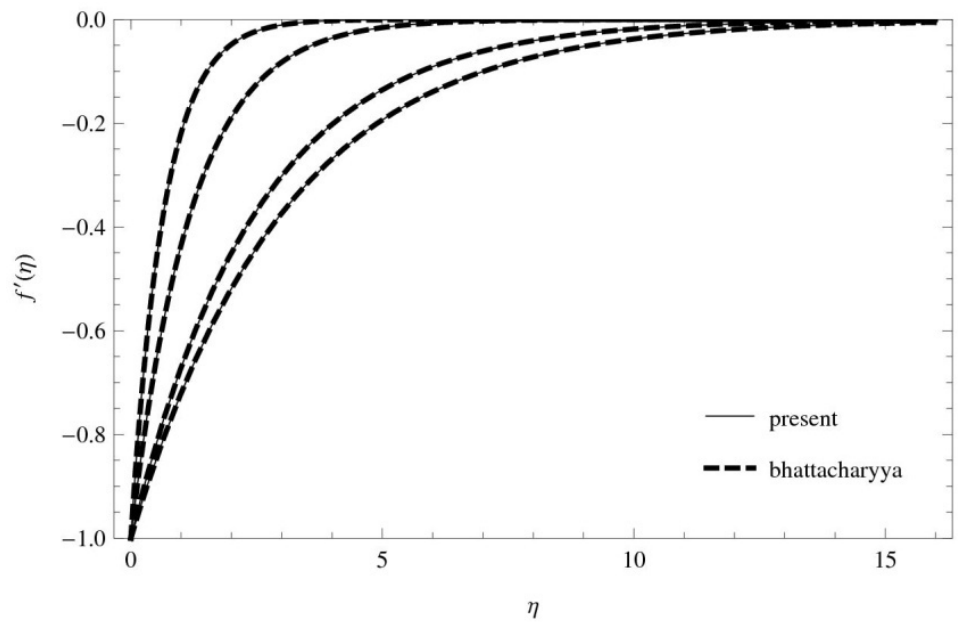

Fig. 10: Comparison of Velocity profiles $f^{\prime}(\eta)$ for shrinking case 


\section{References}

[1] G. Makanda, S. Shaw and P. Sibanda, Diffusion of chemically reactive species in Casson fluid flow over an unsteady stretching surface in porous medium in the presence of a magnetic field, Mathematical Problems in Engineering, 2014, (2014), Article Id 724596.

[2] K. Ramesh and M. Devakar, Some analytical solutions for flows of Casson fluid with slip boundary conditions, Ain Shams Engineering Journal, 6, (2015), 967-975.

[3] D. Mythili and R. Sivaraj, Influence of higher order chemical reaction and non-uniform heat source/sink on Casson fluid flow over a vertical cone and flat plate, Journal of Molecular Liquids, 216, (2016), 466-475.

[4] A.K.A. Hakeem, P. Renuka, N.V. Ganesh, R. Kalaivanan and B. Ganga, Influence of inclined Lorentz forces on boundary layer flow of Casson fluid over an impermeable stretching sheet with heat transfer, Journal of Magnetism and Magnetic Materials, 401, (2016), 354-361.

[5] Z. Abbas, M. Sheikh and S.S. Motsa, Numerical solution of binary chemical reaction on stagnation point flow of Casson fluid over a stretching/shrinking sheet with thermal radiation, Energy, 95, (2016), 12-20.

[6] H.R. Kataria, H.R. Patel, Radiation and chemical reaction effects on MHD Casson fluid flow past an oscillating vertical plate embedded in porous medium, Alexandria Engineering Journal, 55, (2016), 583-595.

[7] C.S.K. Raju, N. Sandeep, V. Sugunamma, M.J. Babu, and J.V.R. Reddy, Heat and mass transfer in magnetohydrodynamic Casson fluid over an exponentially permeable stretching surface, Engineering Science and Technology, an International Journal, 19, (2016), 45-52.

[8] K. Bhattacharyya, T. Hayat and A. Alsaedi, Exact solution for boundary layer flow of Casson fluid over a permeable stretching/shrinking sheet, Zeitschrift für Angewandte Mathematik und Mechanik, 94, (2014), 522 - 528.

[9] S. Pramanik, Casson fluid flow and heat transfer past an exponentially porous stretching surface in presence of thermal radiation, Ain Shams Engineering Journal, 5, (2014), 205-212.

[10] D. Mythili and R. Sivaraj, Influence of higher order chemical reaction and non-uniform heat source/sink on Casson fluid flow over a vertical cone and flat plate, Journal of Molecular Liquids, 216, (2016), 466-475.

[11] S. Mansur and A. Ishak, Unsteady boundary layer flow of a nanofluid over a stretching/shrinking sheet with a convective boundary condition, Journal of the Egyptian Mathematical Society, Article in press, (2016), 1-6.

[12] B. Mohanty, S.R. Mishra and H.B. Pattanayak, Numerical investigation on heat and mass transfer effect of micropolar fluid over a stretching sheet through porous media, Alexandria Engineering Journal, 54, (2015), 223-232.

[13] W. F. Hughes and J. A. Brighton, Dinámica De Los Fluidos, McGraw-Hill, (1967).

[14] U. Filobello-Nino, H. Vazquez-Leal, Y. Khan, A. Perez-Sesma, A. Diaz-Sanchez and A. Herrera-May, A handy exact solution for flow due to a stretching boundary with partial slip, Revista Mexicana de F'1sica E, 59, (2013), 51-55. 